\title{
Laser Material Processing Lead to Nanotoxicity
}

\section{Canan Kurşungöz ${ }^{1,2}$, Sadık Taşkın Taş3 ${ }^{3}$ Barış Alten ${ }^{3}$, Metin Yeşiltepe $^{3}$, M. Yıldırım Sara ${ }^{3}$, Mustafa F. Sargon ${ }^{4}$, Bülend Ortaç ${ }^{1,2}$}

\author{
${ }^{1}$ Materials Science and Nanotechnology Department, Bilkent University, Ankara 06800, Turkey \\ ${ }^{2}$ National Nanotechnology Research Center, UNAM, Bilkent University, Ankara 06800, Turkey \\ ${ }^{3}$ Department of Medical Pharmacology, Hacettepe University, Ankara 06100, Turkey \\ ${ }^{4}$ Anatomy Department, Hacettepe University, Ankara 06100, Turkey
}

Laser ablation is frequently used in the industry in cutting and drilling processes of materials. During these processes, nanoparticles are produced in the air as byproducts [1]. Since these nanoparticles will accumulate in the environment, the workers in that place are exposed to those nanoparticles. It was shown that when animals are exposed to nanoparticles via inhalation, nanoparticles enter systemic circulation via alveolar epithelium. Nanoparticles travel through the systemic circulation to reach vital organs like heart, brain and liver [2]. Those nanoparticles lead to disruption of a number of cellular signaling pathways and consequently result in cellular toxicity [3].

We investigated the body distribution of copper, tin and aluminum NPs (CuNP, SnNP and AlNP) generated in laser material processing environment similar to that of real life human exposure in rats. We have first characterized those NPs by SEM, TEM and EDAX techniques. The rats were exposed to NPs for 3 months (Figure 1a). For the first time, we have shown that exposure to NPs caused accumulation in various organs (such as lung, kidney, liver and spleen) during real time laser ablation process. Following SnNP exposure of rats, NPs up to $400 \mathrm{ppb}$ were detected in lungs by ICP-MS (Figure 1b, c). We have also showed that CuNP, SnNP and AlNP produced with pulsed laser ablation method lead to cellular toxicity and cell death in a dose dependent manner in SH-SY5Y neuron cell line.

Our data indicated that material processing by laser leads to the generation of nanoparticles in the work environment. Chronic exposure of workers to nanoparticles will result in end-organ accumulation and consequently may cause irreversible organ damage and serious health problems. This project is supported by TÜBİTAK (Project No: 113S223).

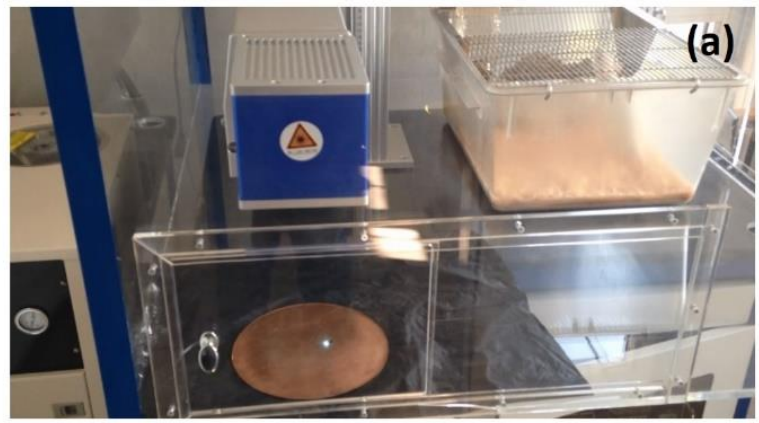

(b)

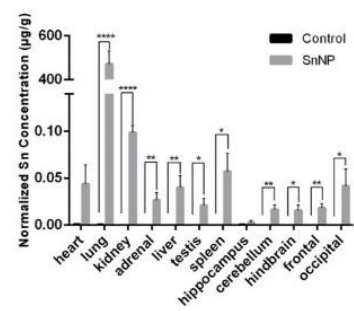

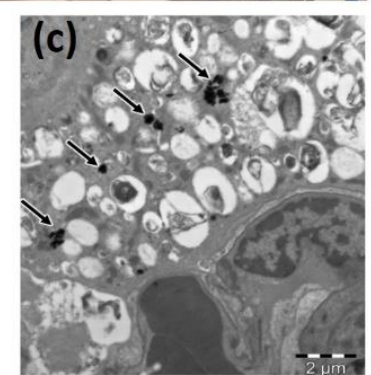

Figure 1: (a) Laser material processing setup and real time exposure system, (b) ICP-MS results of SnNP distribution in various organs, (c) Representative TEM image of lung sample from SnNP exposed rat.

\section{References:}

[1] S. Barcikowski, A. Hahn, J. Walter. "Health risks of nanoparticulate emissions during femtosecond and picosecond pulsed laser machining" SPIE LASE,720109 (2009).

[2] Elsaesser, Andreas, and C. Vyvyan Howard. "Toxicology of nanoparticles." Advanced drug delivery reviews, 64,129 (2012).

[3] Block, Michelle L., and Lilian Calderón-Garcidueñas. "Air pollution: mechanisms of neuroinflammation and CNS disease." Trends in neurosciences 32, 506-516 (2009). 